NASA Technical Memorandum 103754

\title{
Designs for the ATDRSS Tri-Band Reflector Antenna
}

Shung-Wu Lee and Martin L. Zimmerman University of Illinois, Urbana-Champaign Urbana, Illinois

and

Gene Fujikawa and G. Richard Sharp

Lewis Research Center

Cleveland, Ohio

Prepared for the

1991 IEEE AP-S International Symposium and URSI Radio Science Meeting London, Ontario, Canada, June 24-28, 1991 


\title{
DESIGNS FOR THE ATDRSS TRI-BAND REFLECTOR ANTENNA
}

\author{
Shung-Wu Lee and Martin L. Zimmerman \\ University of Illinois, Urbana-Champaign \\ Urbana, Illinois 61801 \\ Gene Fujikawa and G. Richard Sharp \\ National Aeronautics and space Administration \\ Lewis Research Center \\ Cleveland, Ohio 44135
}

NASA's Tracking and Data Relay Satellite System (TDRSS) presently provides a vital link in space communications. The TDRSS satellites substantially increase Earth-to-space link availability and provide a near continuous exchange of information. A single TDRSS satellite can transmit and receive high-data-rate information to and from low earth orbiting spacecraft via two single access (SA) reflector antennas. These steerable SA antennas can provide simultaneous S-band and Ku-band communications with one spacecraft at a time. Communications to and from orbiting spacecraft can also be accomplished via an S-band multiple access phased array antenna, though at much lower data rates. A separate space-to-ground link antenna operating at Ku-band provides communications between a TDRSS satellite and the TDRSS White Sands Ground Terminal in New Mexico.

In order to provide additional bandwidth for increased communications demand, the advanced TDRSS, or ATDRSS project has been proposed and is scheduled for launch in 1997. As conceived, the ATDRSS satellites will incorporate Ka-band capability in the SA reflector antennas, in addition to the S-band and Ku-band services. Therefore, in order to meet these future requirements, the development of a tri-band reflector antenna for ATDRSS is critical.

There are two approaches to designing a tri-band reflector antenna. The first approach utilizes multi-band feeds (e.g. one feed for S-band and another for Ku-band and $\mathrm{Ka-band).} \mathrm{However,} \mathrm{it} \mathrm{is} \mathrm{difficult} \mathrm{to} \mathrm{optimize} \mathrm{the} \mathrm{antenna} \mathrm{performance} \mathrm{for} \mathrm{a}$ single feed at more than one frequency. The task is made more difficult by the broad bandwidth required for the Ka-band (21\%).

In this paper, we use a second approach, in which three separate feeds are used. This allows each feed to be optimized for a single band. The feeds are isolated by means of frequency selective surfaces (FSS). The FSS will transmit certain frequencies while reflecting others. Conventionally, most Fsss are designed to be used in two frequency bands. The challenge is to design an Fss that works for three bands (e.g. transmitting two bands and reflecting a third). Two reflector antenna configurations are presented below, an offset single reflector (fig. 1) and a symmetric shaped dual reflector (fig. 2). The advantages of each design are presented in table I.

FSS designs have been developed that will provide the necessary transmission and reflection characteristics. Using these designs, far-field patterns have been computed for the above antenna systems, including losses due to the FSS effects. In general, the FSS losses are only a few tenths of a $\mathrm{dB}$. One of the worst cases for the offset configuration, at the upper edge of the wide Ka band, is shown in figure 3. Losses here are only $0.4 \mathrm{~dB}$, based on theoretical calculations.

The offset fed single reflector antenna configuration has been selected for integration into proposed ATDRS designs. The offset design uses a solid reflector and planar FSSs, which can be accurately modeled by existing computer programs using 
modal analysis. The offset fed single access antenna geometry shown in figure 1 has been adapted for a conceptual spacecraft design. Some of the assumptions for this design are (1) an Atlas Centaur launch vehicle, (2) an Advanced Communications Technology satellite (ACTS) size spacecraft body and ACTS type solar arrays and (3) the S-band amplifier can be located in the spacecraft body and the Ku- and Ka-band equipment can be located in the antenna arms near the feed horns.

CADAM drawings have been completed for the design concept and a 1/13th scale model has been constructed, employing this offset-fed antenna configuration. Figure 4 is a photograph of the spacecraft model in the launch ready state. Figure 5 shows the spacecraft model with both offset fed antennas fully deployed. This design exceeds the original ATDRSS single access antenna pointing requirements in that it allows a full $360^{\circ}$ offset antenna scanning capability for Lunar access when using both antennas.

TABLE I. - DESIGN SUMMARY

\begin{tabular}{|c|l|l|}
\hline Main reflector & FSS & \multicolumn{1}{|c|}{ Advantages } \\
\hline $\begin{array}{c}\text { Parabolic, offset } \\
\text { figure 1 }\end{array}$ & 2 Planar & $\begin{array}{l}\text { Planar Fss only } \\
\text { Smaller diameter main reflector } \\
(12.5 \mathrm{ft}) \\
\text { Solid reflector, not mesh }\end{array}$ \\
$\begin{array}{c}\text { Shaped, symmetrical } \\
\text { figure 2 }\end{array}$ & 1 Planar and 1 curved & $\begin{array}{c}\text { Smaller volume (shorter focal } \\
\text { length) } \\
\text { Similar to existing TDRS design }\end{array}$ \\
\hline
\end{tabular}




\begin{tabular}{|l|l|l|l|}
\hline \multicolumn{1}{|c|}{ Gain/loss } & $\begin{array}{c}\text { S-band } \\
2.2 \mathrm{GHz}\end{array}$ & $\begin{array}{c}\text { Ku-band } \\
14.9 \mathrm{GHz}\end{array}$ & $\begin{array}{c}\text { Ka-band } \\
25.25 \mathrm{GHz}\end{array}$ \\
\hline $\begin{array}{l}\text { Ideal gain } \\
\text { (D=150 in.) }\end{array}$ & $38.87 \mathrm{~dB}$ & $55.48 \mathrm{~dB}$ & $60.06 \mathrm{~dB}$ \\
\hline Spillover/illumination & -1.38 & -1.36 & -1.33 \\
Blockage & -0.05 & -0.0 & -0.0 \\
FSS & -0.30 & -0.10 & -0.80 \\
Reflector surface & -0.05 & -0.17 & -0.73 \\
Radome & -0.05 & -0.0 & -0.0 \\
Feed loss & -0.4 & -0.5 & -0.7 \\
\hline Total loss & -2.23 & -2.13 & -3.56 \\
Efficiency & $60 \%$ & $61 \%$ & $44 \%$ \\
Net gain & $36.6 \mathrm{~dB}$ & $53.3 \mathrm{~dB}$ & $56.5 \mathrm{~dB}$ \\
\hline
\end{tabular}

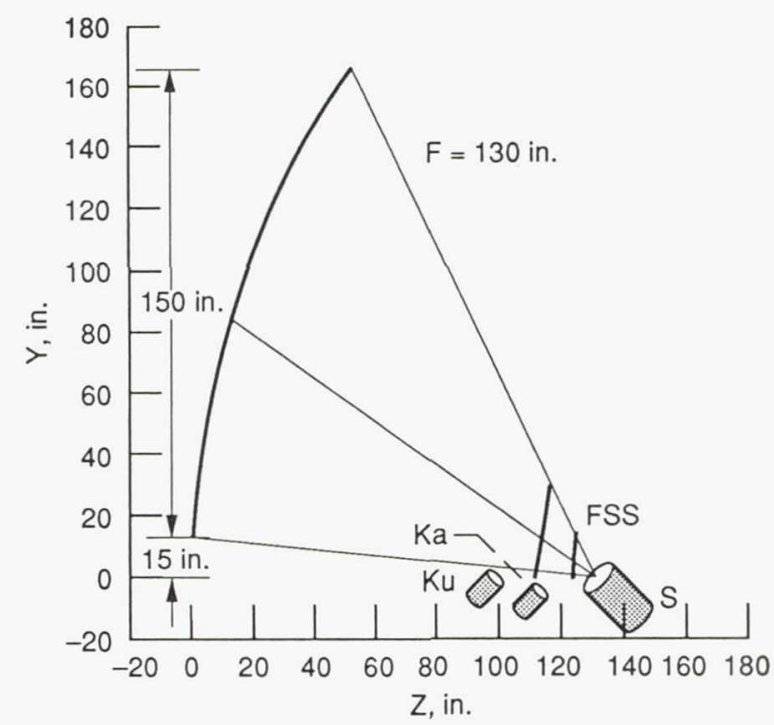

Figure 1.-Offset single reflector tri-band configuration for ATDRSS with two planar FSS. 


\begin{tabular}{|l|l|l|l|}
\hline \multicolumn{1}{|c|}{ Gain/loss } & \multicolumn{1}{c|}{$\begin{array}{c}\text { S-band } \\
2.2 \mathrm{GHz}\end{array}$} & $\begin{array}{c}\text { Ku-band } \\
14.9 \mathrm{GHz}\end{array}$ & $\begin{array}{c}\text { Ka-band } \\
25.25 \mathrm{GHz}\end{array}$ \\
\hline $\begin{array}{l}\text { Ideal gain } \\
\text { (D=168 in.) }\end{array}$ & $39.85 \mathrm{~dB}$ & $56.47 \mathrm{~dB}$ & $61.05 \mathrm{~dB}$ \\
\hline $\begin{array}{l}\text { Spillover/block } \\
\text { illumination }\end{array}$ & -2.0 & -0.53 & -0.6 \\
$\begin{array}{l}\text { Planar FSS } \\
\text { Additional loss for } \\
\text { curved FSS }\end{array}$ & -0.50 & -0.50 & -0.70 \\
$\begin{array}{l}\text { Reflector surface } \\
\text { (mesh) }\end{array}$ & -0.20 & -0.20 & -0.20 \\
$\begin{array}{l}\text { Radome } \\
\text { Feed loss }\end{array}$ & -0.05 & -0.68 & -2.3 \\
Feed line run & -0.05 & -0.0 & -0.0 \\
\hline Total loss & -0.4 & -0.5 & -0.7 \\
Efficiency & -3.60 & -0.12 & -0.37 \\
Net gain & $44 \%$ & $56 \%$ & -4.83 \\
\hline
\end{tabular}
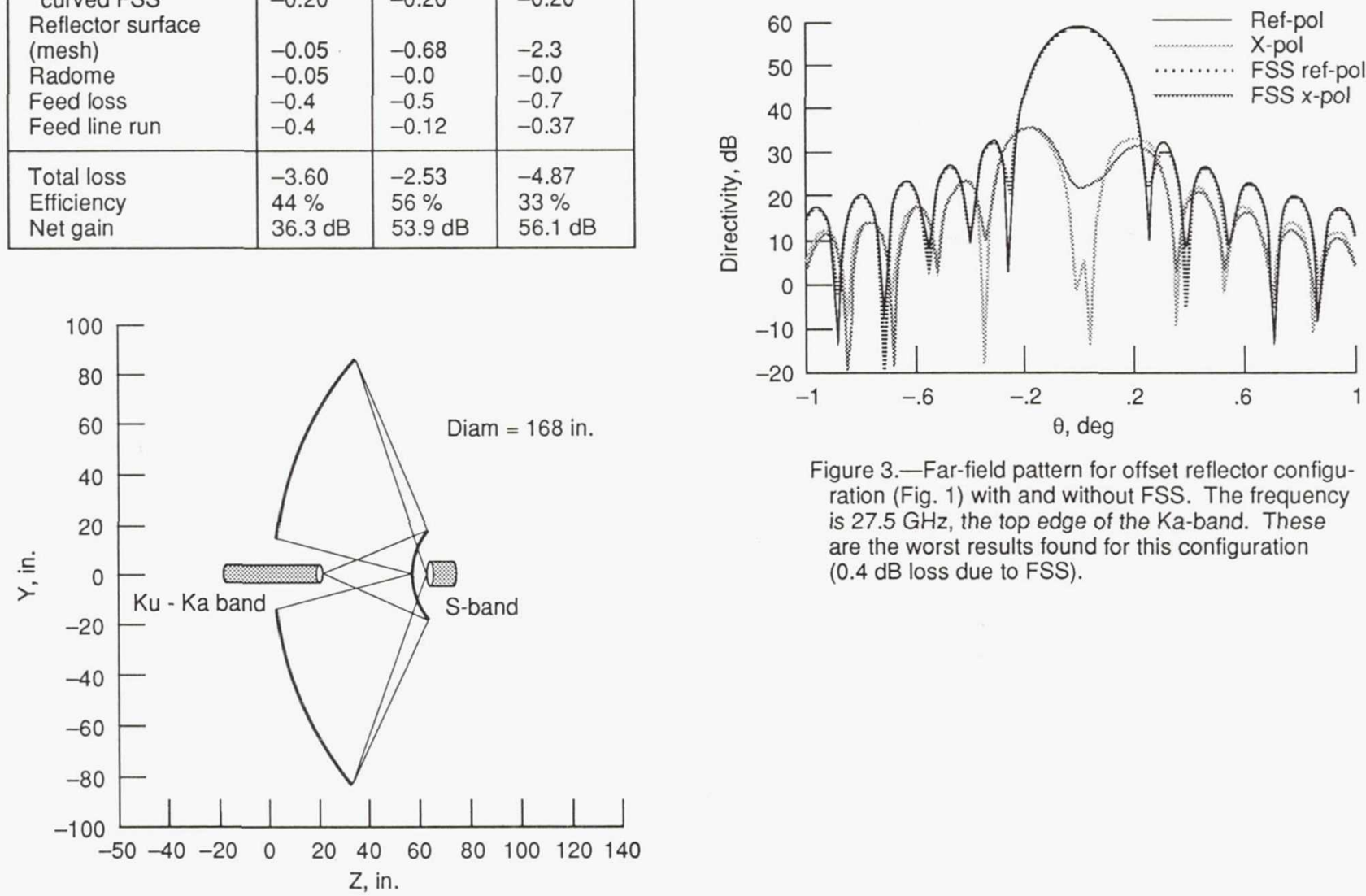

Figure 3.-Far-field pattern for offset reflector configuration (Fig. 1) with and without FSS. The frequency is $27.5 \mathrm{GHz}$, the top edge of the Ka-band. These are the worst results found for this configuration ( $0.4 \mathrm{~dB}$ loss due to $\mathrm{FSS}$ ).

Figure 2.-Symmetric dual-shaped reflector configuration for ATDRSS. The subreflector is an FSS and a planar FSS (not shown) separates the Ka-band and Ku-band. 


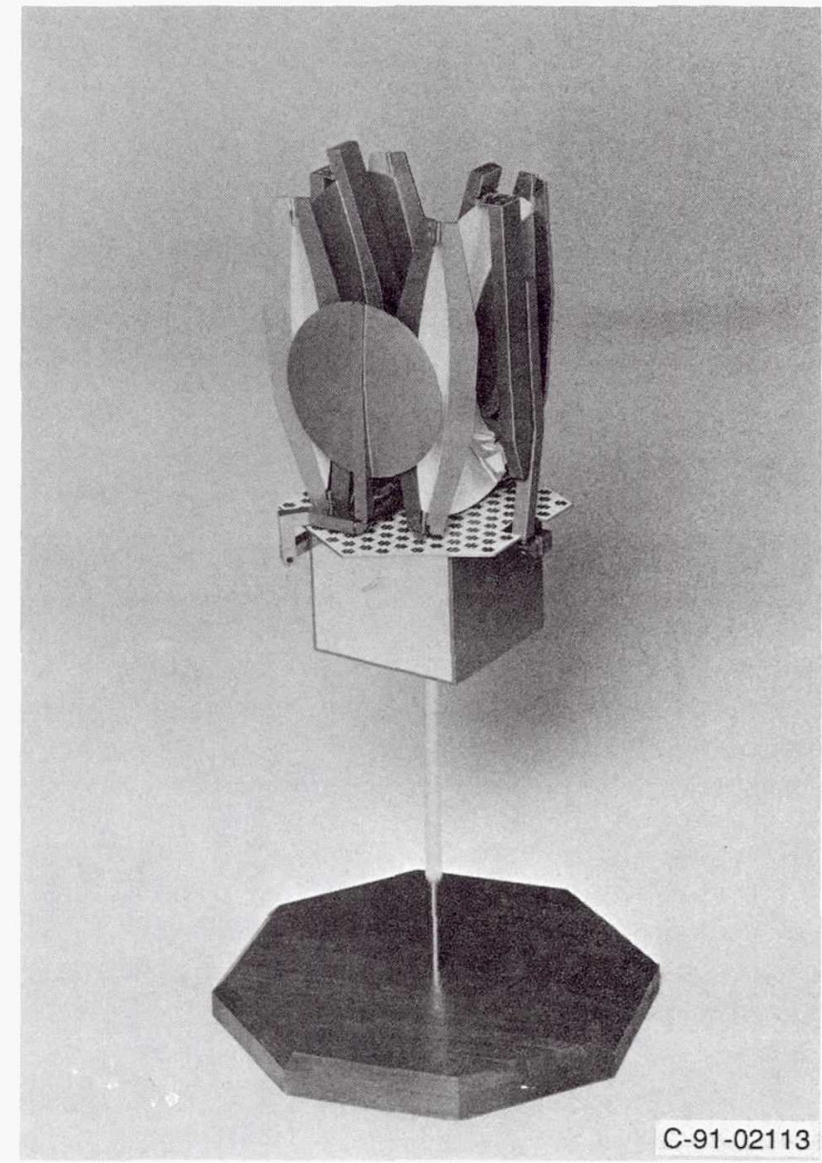

Figure 4.-1/13th scale model of ATDRS design concept incorporating offset-fed single reflector antennas in launch configuration.

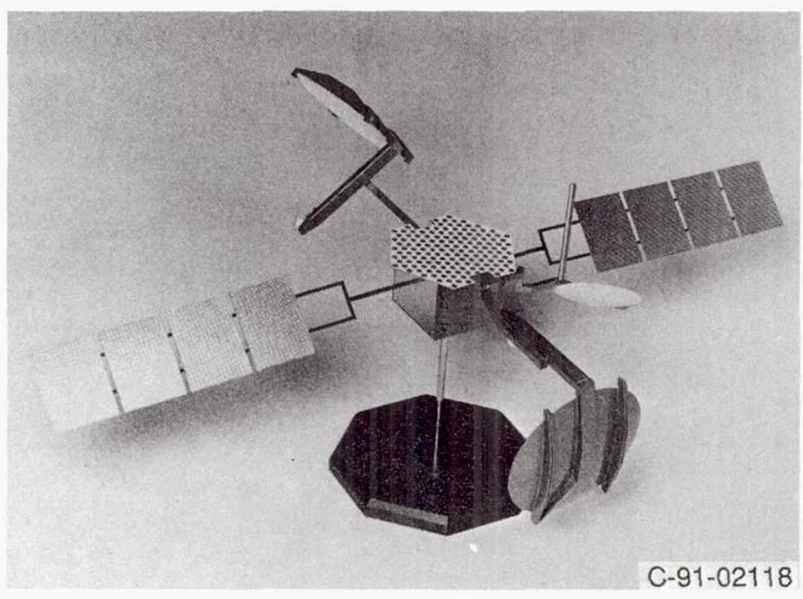

Figure 5.-Fully deployed 1/13th scale ATDRS model with offsetfed antennas demonstrating a full $360^{\circ}$ spherical scan capability. 


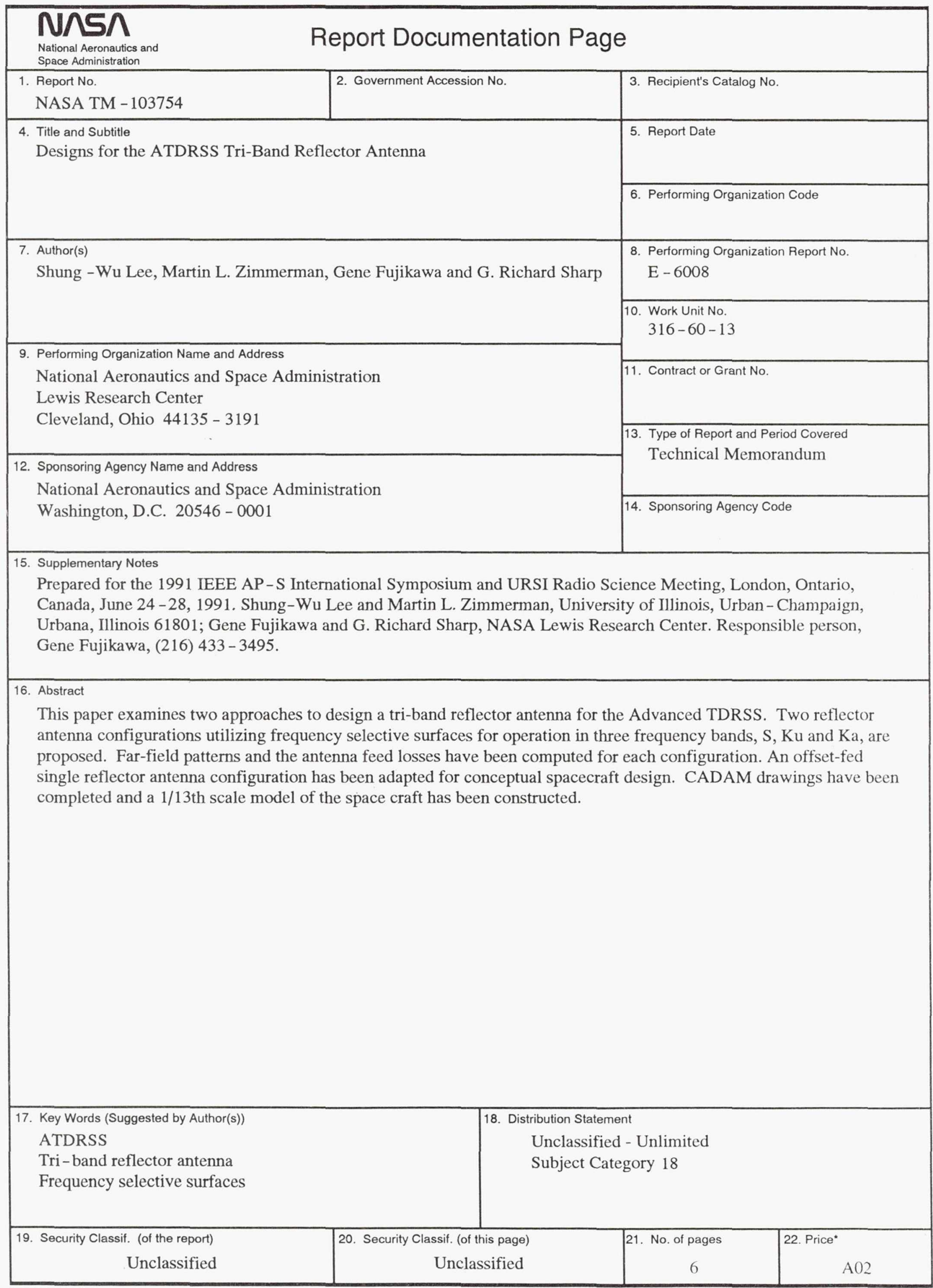

